\title{
Melanoma anal, importancia del diagnóstico diferencial con la enfermedad anal benigna
}

\author{
David Martínez-Ramos ${ }^{1}$, Rafael García-Calvo ${ }^{1}$, Laura Simón-Monterde ${ }^{2}$, María Jesús Nicolau-Ribera $^{3}$
}

Palabras clave: melanoma; neoplasias del ano; membrana mucosa; diagnóstico diferencial; oncología quirúrgica.

\section{Resumen}

El melanoma anorrectal, descrito inicialmente por Moore en 1857, es un tumor infrecuente y agresivo, cuyo pronóstico suele ser infausto, con una supervivencia a los cinco años menor de $20 \%$. La principal importancia de este tumor radica en que suele presentarse como una tumoración anorrectal o producir sangrado, o rectorragia, por lo que el diagnóstico diferencial con la enfermedad anal benigna resulta crucial, pues el pronóstico estará condicionado por el estadio en el momento del diagnóstico.

Se presenta el caso de una mujer de 70 años que consultó por sangrado rectal y cuyo diagnóstico inicial fue de trombosis hemorroidal. Tras el diagnóstico de melanoma anorrectal y el estudio de extensión negativo para metástasis, se practicó una amputación abdómino-

1 Servicio de Cirugía General y Aparato Digestivo, Hospital Universitario General de Castellón, Castellón, España

2 Servicio de Urgencias, Consorcio Hospitalario Provincial de Castellón, Castellón, España

3 Servicio de Anatomía Patológica, Hospital Universitario General de Castellón, Castellón, España

Fecha de recibido: 12 de octubre de 2016

Fecha de aprobación: 21 de noviembre de 2016

Citar como: Martínez-Ramos D, García-Calvo R, Simón-Monterde L, Nicolau-Ribera MJ. Melanoma anal, importancia del diagnóstico diferencial con la enfermedad anal benigna. Rev Colomb Cir. 2017;32:330-34. perineal según la técnica de Miles. Tras nueve años de seguimiento, la paciente se encuentra libre de enfermedad.

\section{Introducción}

El melanoma es una neoplasia que se produce por un crecimiento incontrolado de los melanocitos, células que se derivan embriológicamente de la cresta neural. La localización más frecuente de los melanomas es en la piel, pero pueden aparecer también en otras localizaciones, como los ojos o en diferentes mucosas, como la gastrointestinal y la genital. De este modo, el melanoma que aparece en las mucosas se ha considerado como una entidad diferente al melanoma cutáneo, debido a su diferente comportamiento biológico y, en general, debido a su peor pronóstico ${ }^{1}$.

El canal anal es el lugar más frecuente de aparición de los melanomas de la mucosa gastrointestinal ${ }^{2}$. Así, aunque representa menos de 0,05 a 4,6 \% de las lesiones anorrectales ${ }^{3,4}$, esta constituye la tercera localización más frecuente del melanoma, después de la piel y los ojos ${ }^{1}$. El melanoma anorrectal, descrito inicialmente por Moore en $1857^{5}$, es un tumor infrecuente y agresivo, cuyo pronóstico suele ser infausto, con una supervivencia a los cinco años menor del $20 \%{ }^{3}$.

La principal importancia de este tumor radica en que suele presentarse como una tumoración anorrectal o mediante sangrado o rectorragia, por lo que el diagnóstico diferencial con la enfermedad anal benigna resulta crucial, pues el pronóstico está condicionado por el estadio en el momento del diagnóstico ${ }^{6,7}$. 
El objetivo de este artículo fue presentar nuestra experiencia en el diagnóstico y tratamiento de una paciente con un melanoma anal y destacar, así, la importancia de un diagnóstico diferencial con la enfermedad anal benigna, especialmente con las hemorroides, dado el carácter nodular y habitualmente pigmentado que pueden presentar ambas lesiones.

\section{Caso clínico}

Se trata de una mujer de 70 años que acudió al Servicio de Urgencias del Hospital Universitario General de Castellón, por presentar una tumoración sobreelevada y de coloración azulada-negruzca, sangrante y de $1 \mathrm{~cm}$ de diámetro, aproximadamente, localizada en la región perianal, de unas tres semanas de evolución (figura 1).

Con el diagnóstico presuntivo inicial de trombosis hemorroidal, fue sometido a hemorroidectomía, bajo anestesia local y regional; no hubo complicaciones y se dio el alta hospitalaria al día siguiente de la intervención.

El estudio anatomopatológico de la pieza de resección quirúrgica reportó la presencia de un melanoma ulcerado, de localización perianal, cuyo máximo espesor de Breslow era de 1,3 $\mathrm{mm}$ y que alcanzaba el margen profundo.

Con este inesperado diagnóstico, se completó el estudio de extensión mediante tomografía computadorizada (TC) toraco-abdómino-pélvica, colonoscopia y biopsia selectiva de ganglios centinelas inguinales de

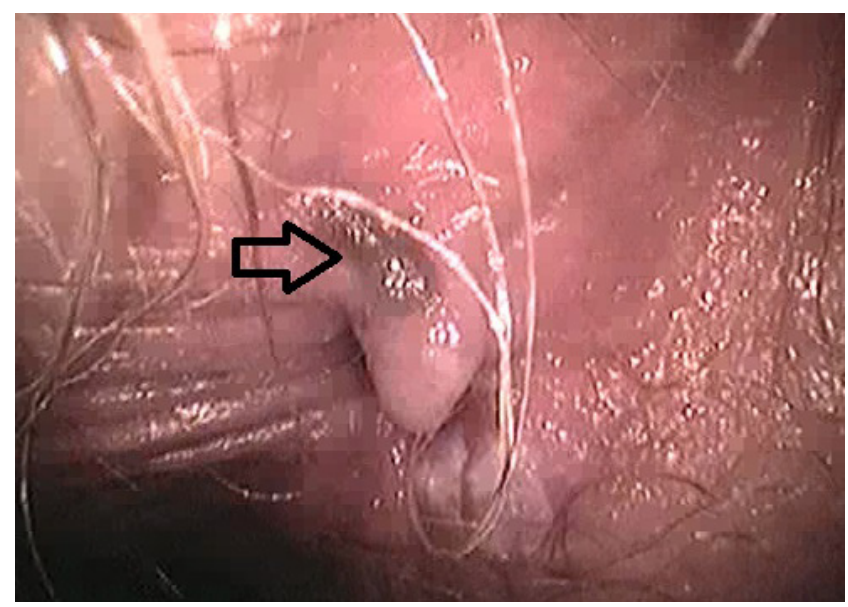

FIgURA 1. Exploración anal en la que se aprecia pequeña hemorroide no complicada y lesión melánica compatible con el melanoma extirpado (flecha). ambos lados. No se practicó PET-TC por no encontrarse disponible en nuestro medio en aquel momento. La TC resultó negativa para metástasis. La colonoscopia demostró múltiples lesiones melánicas en la mucosa rectal, compatibles con metástasis o extensión local de la enfermedad (figura 2). Los tres ganglios inguinales centinelas estudiados, dos izquierdos y uno derecho, resultaron negativos para neoplasia maligna.

Con estos resultados, se decidió completar el tratamiento quirúrgico con intención curativa, procediéndose a una amputación abdómino-perineal, según la técnica descrita por Miles. El periodo posoperatorio nuevamente transcurrió sin complicaciones. El estudio anatomopatológico de la pieza de resección recto-sigmoidea confirmó la presencia de un melanoma nodular de localización perianal, con un espesor máximo de Breslow de $5 \mathrm{~mm}$; asimismo, infiltración de la mucosa rectal, que alcanzaba $4 \mathrm{~mm}$ de profundidad. No hubo metástasis en los tres ganglios analizados. Los márgenes quirúrgicos estaban libres de neoplasia (figura 3).

Ante la presencia de un melanoma de alto riesgo, se propuso tratamiento adyuvante con interferón, según el esquema de Kirwood, completando un total de 20 sesiones. Posteriormente, se inició tratamiento de mantenimiento durante un año con interferón, 15 millones de UI por vía subcutánea tres veces por semana.

En el seguimiento, persistió libre de enfermedad hasta ocho años más tarde, cuando en una TC de control se observó un nódulo pulmonar en el lóbulo medio, hiper-

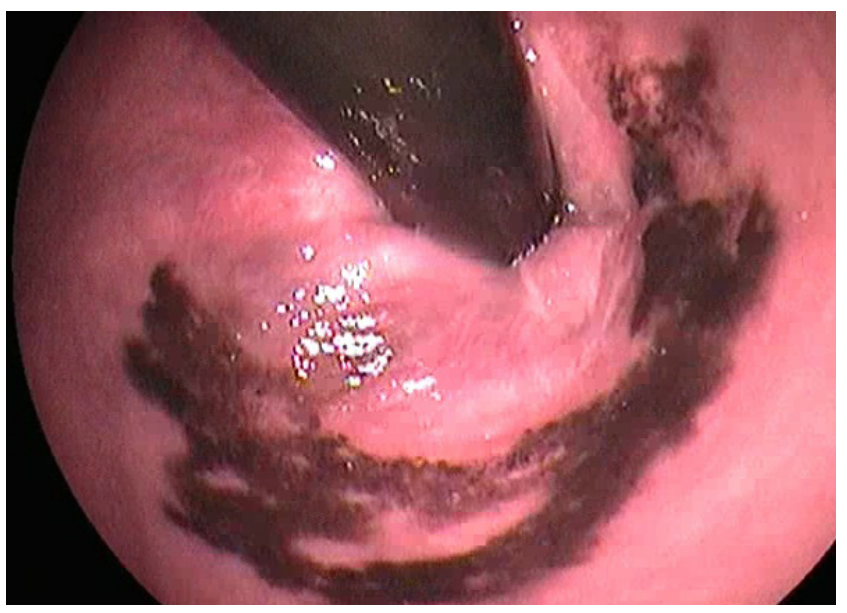

FIGURA 2. Colonoscopia en retrovisión, donde se aprecia la extensión a canal rectal de las lesiones de melanoma. 


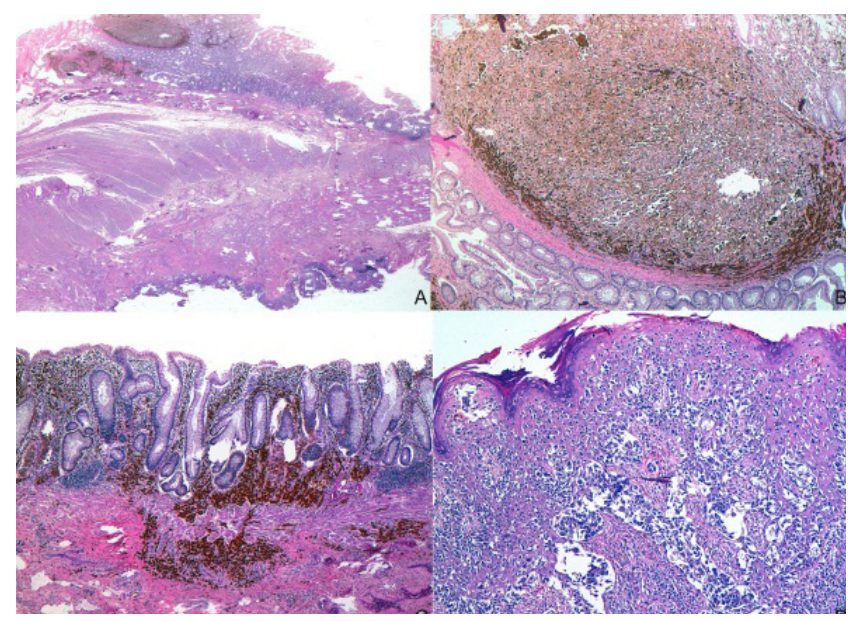

Figura 3. Estudio anatomopatológico. A. Agregado nodular de células pigmentadas atípicas con crecimiento intramucoso. Hematoxilina y eosina, lupa. B. La misma imagen con mayor aumento. Hematoxilina y eosina, 10X. C. Melanoma que infiltra más allá de la muscularis mucosa, con patrón de pequeños nidos y células sueltas hiperpigmentadas, a distancia de la lesión nodular descrita, en otra zona de la mucosa del colon. Hematoxilina y eosina, $10 \mathrm{X}$. D. Crecimiento pagetoide de las células melánicas malignas en el epitelio escamoso anorrectal. Hematoxilina y eosina, $10 \mathrm{X}$

metabólico en la PET-TC, con características sugestivas de neoplasia maligna. La resonancia magnética también demostró la presencia de un nódulo pulmonar de $20 \mathrm{x}$ $16 \mathrm{~mm}$, levemente hiperintenso en $\mathrm{T}_{1}$, sin aumento de la intensidad de la señal en las imágenes potenciadas en $\mathrm{T}_{2}$, compatible con metástasis de melanoma.

Ante la presencia de un nódulo pulmonar sospechoso caracterizado como metástasis única, se practicó su resección quirúrgica a través de una toracotomía derecha, mediante cuña pulmonar grapada en el lateral, sin que se presentaran complicaciones. El estudio anatomopatológico confirmó la presencia de una metástasis de melanoma de $2 \mathrm{~cm}$ que infiltraba la pleura visceral y llegaba a la superficie. En el margen de resección quirúrgica no había infiltración tumoral y el parénquima pulmonar circundante mostraba leves cambios inespecíficos.

En el control de seguimiento, seis meses después de la metastasectomía y nueve años después de la extirpación del tumor primario, la paciente se encontraba libre de enfermedad.

\section{Discusión}

El melanoma anal se presenta de manera típica como una masa rectal o con un sangrado rectal. Suele tratarse de lesiones con pequeñas zonas pigmentadas, con úlceras profundas o una masa polipoide o exofítica ${ }^{8}$, aunque se han comunicado casos de melanomas amelanóticos en la región anal ${ }^{9,10}$. Por ello, en muchas ocasiones puede confundirse este agresivo tumor con un proceso anal benigno, como hemorroides, repliegues cutáneos, papilomas, etc., como ocurrió inicialmente en esta paciente.

Hace unos años, publicamos la escasa exactitud del diagnóstico de enfermedad anal benigna en nuestro medio ${ }^{11}$, por lo que resulta de especial importancia conocer casos como el aquí presentado, pues es muy probable que el retraso en el diagnóstico produzca empeoramiento del pronóstico ${ }^{3,12}$. De hecho, hasta $38 \%$ de los casos se presentan con metástasis a distancia en el momento del diagnóstico ${ }^{13}$.

El melanoma anorrectal es relativamente insensible a la radioterapia y a la quimioterapia, aunque se han comunicado recientemente algunas mejorías parciales, sobre todo en tratamientos paliativos o de rescate para cirugía ${ }^{14}$. Por todo ello, el tratamiento de elección es la resección quirúrgica, aunque siguen siendo temas controvertidos el tipo de cirugía y la extensión de la resección.

Debido a que es un tumor tan infrecuente, no existen ensayos clínicos controlados, y los trabajos con mayor número de pacientes son series no aleatorizadas. Así, por ejemplo, en un estudio llevado a cabo en el Instituto Nacional del Cáncer de Milán de 31 pacientes ${ }^{15}$, no consiguieron demostrar diferencias entre la resección abdómino-perineal, la resección rectal con anastomosis coloanal o la resección local amplia. Brady, et al. ${ }^{2}$, comunicaron su experiencia con 71 pacientes tratados con resección abdómino-perineal, escisión local amplia, o biopsia, fulguración o ambas. Aunque no encontraron diferencias estadísticamente significativas en la supervivencia, la recurrencia local fue del $8 \%$ en el grupo con amputación abdómino-perineal y, de $20 \%$, en el tratado localmente; por tanto, recomendaron la amputación abdómino-perineal como tratamiento de elección en estos casos. Otros autores ${ }^{12}$ consideran que la resección local ofrece mejores resultados funcionales, aunque no se demuestran diferencias significativas en la supervivencia global ${ }^{8}$.

En una revisión sistemática llevada a cabo por Kanaan, et al. ${ }^{16}$, sobre cerca de 700 pacientes, no se encontraron diferencias en la supervivencia global entre la ampu- 
tación abdómino-perineal y la escisión local amplia, aunque se admitió que existía demasiada variabilidad en la estadificación local y ganglionar para poder hacer recomendaciones definitivas.

Así las cosas, de acuerdo con las conclusiones de Nam, et al. ${ }^{4}$, debería individualizarse el tratamiento según los casos, pues no existe suficiente evidencia científica para recomendar uno u otro tratamiento quirúrgico. Lo más importante, eso sí, es obtener márgenes de resección libres de tumor ${ }^{17}$. En el presente caso, la extensión de las lesiones desde el margen anal hasta el canal rectal, hizo necesaria la resección mediante amputación abdómino-perineal.

Con respecto a la linfadenectomía inguinal, no se ha demostrado que mejore el pronóstico $y$, además, conlleva elevada morbilidad. Por ello, técnicas como la biopsia selectiva de ganglio centinela parecen prometedoras, aunque la experiencia sigue siendo limitada ${ }^{18}$. En el presente caso, esta técnica fue realizada de manera adecuada, lo que permitió la correcta estadificación ganglionar.

Recientemente, se han comunicado también buenos resultados con el abordaje laparoscópico para el tratamiento del melanoma anorrectal ${ }^{5,19}$. Aunque prometedores, estos resultados deben ser tomados con precaución, puesto que se trata de casos aislados en grupos de trabajo muy especializados.

Aunque usualmente se habla de melanoma anorrectal, en el estudio de Bello, et al. ${ }^{20}$, se demostraron algunas diferencias en función de la localización del melanoma en el recto o en el ano. De esta forma, según estos resultados, las lesiones localizadas proximalmente a la línea dentada se presentarían en un estadio más avanzado, probablemente por un retraso en el diagnóstico. Las lesiones distales a esta línea presentan mayor recurrencia linfática, lo que correspondería a diferencias en el sistema de drenaje ganglionar. Sin embargo, en ambos grupos el pronóstico fue infausto, con escasa supervivencia a largo plazo. De hecho, la supervivencia global para ambos grupos a los cinco años es menor de $20 \%$ en la mayoría de trabajos ${ }^{12}$, aunque existen casos comunicados con una supervivencia mayor ${ }^{21}$ como el aquí presentado, cuya supervivencia está siendo anormalmente elevada.

Con respecto a los tratamientos adyuvantes en el melanoma, este tumor muestra más susceptibilidad al tratamiento inmunológico que los tumores de otra estirpe y, por ello, ha sido objeto de una gran variedad de ensayos basados en inmunoterapia, con algunos resultados esperanzadores. En el caso concreto del melanoma anal, su uso puede considerarse, según Reina, et al. ${ }^{18}$, como experimental. Además, el melanoma anorrectal no mejora con la quimioterapia actual. También, es dudosa la utilidad de la radioterapia para el tratamiento del melanoma, debido a la radiorresistencia de este tumor ${ }^{18}$.

En resumen, el melanoma anorrectal es una entidad infrecuente y de pronóstico infausto. No obstante, es necesario mantener un alto índice de sospecha para poder hacer un diagnóstico precoz que permita un tratamiento quirúrgico óptimo, para mejorar los resultados en cuanto a supervivencia y recurrencia local.

\title{
Anal melanoma, the importance of differential diagnosis with benign anal pathology
}

\begin{abstract}
Anorectal melanoma was first described by Moore in 1857. Is an uncommon and aggresive tumor, with inauspicious prognosis. Five-year survival rates are less than 20\%. The main important feature of this tumor is that it usually manifests as a rectal tumor or rectal bleeding; therefore, a diferential diagnosis with benign anal conditions is of utmost importance. Prognosis is conditioned by tumor stage at diagnosis.

We present a 70-year-old woman with rectal bleeding with an initial diagnosis of hemorrhoidal trombosis. With the diagnosis of rectal melanoma without distant metastasis, an abdominoperineal resection (Miles operation) was performed. After 9-years follow-up, she is disease free.
\end{abstract}

Key words: Melanoma; anus neoplasms; mucous membrane; diagnosis, differential; surgical oncology. 


\section{Referencias}

1. Carcoforo P, Raiji MT, Palini GM, Pedriali M, Maestroni U, Soliani G, et al. Primary anorectal melanoma: An update. J Cancer. 2012;3:449-53.

2. Brady MS, Kavolius JP, Quan SH. Anorectal melanoma. A 64year experience at Memorial Sloan-Kettering Cancer Center. Dis Colon Rectum. 1995;38:146-51.

3. Damodaran O, Morgan A, Mendelsohn G. Primary malignant melanoma in the anorectum: An uncommon cancer. $\mathrm{N} Z \mathrm{Med}$ J. 2008;121:66-8.

4. Nam S, Kim CW, Baek SJ. The clinical features and optimal treatment of anorectal malignant melanoma. Ann Surg Treat Res. 2014;87:113-7.

5. Han J, Shi C, Dong X. Laparoscopic abdomino-perineal resection for patients with anorectal malignant melanoma: A report of 4 cases. J Biomed Res. 2013;27:1-6.

6. Martínez-Hernández-Magro P, Villanueva-Sáenz E, ChávezColunga LB. Melanoma maligno anal. Reporte de casos y revisión de la literatura. Rev Gastroenterol Mex. 2009;74:39-44.

7. Podnos YD, Tsai NC, Smith D, Ellenhorn JD. Factors affecting survival in patients with anal melanoma. Am Surg. 2006;72: 917-20.

8. Lombana LJ, Maciel V, Centeno M, Valencia A. Melanoma anorrectal: resección local amplia versus resección abdominoperineal. Univ Méd. 2005;46:56-8.

9. Núñez-Garbín A, Córdova-Pantoja C, Patiño-Ascona S, Santillana-Callirgos J. Melanoma anal amelanótico. Reporte de caso. Rev Colomb Gastroenterol. 2013;28:359-62.

10. Trzcinski R, Kujawski R, Mik M, Sygut A, Dziki L, Dziki A. Malignant melanoma of the anorectum-a rare entity. Langenbecks Arch Surg. 2010;395:757-60.

11. Martínez-Ramos D, Nomdedéu-Guinot J, Artero-Sempere R, Escrig-Sos J, Gibert-Gerez J, Alcalde-Sánchez M, et al. Estudio prospectivo para evaluar la precisión diagnóstica en patología anal benigna en Atención Primaria. Aten Primaria. 2009;41: 207-12.

12. Heeney A, Mulsow J, Hyland JM. Treatment and outcomes of anorectal melanoma. Surgeon. 2011;9:27-32.
13. Ibnian AM, Nagaraja V, Eslick GD, Kalanta JS. Rectal melanoma with multiple metastases: A rare and aggressive tumor. Rare Cancers Ther. 2014;2:11-6.

14. Moozar KL, Wong CS, Couture J. Anorectal malignant melanoma: Treatment with surgery or radiation therapy, or both. Can J Surg. 2003;46:345-9.

15. Belli F, Gallino GF, Lo Vullo S, Mariani L, Poiasina E, Leo E. Melanoma of the anorectal region: The experience of the National Cancer Institute of Milano. Eur J Surg Oncol. 2009;35: 757-62.

16. Kanaan Z, Mulhall A, Mahid S, Torres ML, McCafferty M, McMasters KM, et al. A systematic review of prognosis and therapy of anal malignant melanoma: a plea for more precise reporting of location and thickness. Am Surg. 2012;78:28-35.

17. Nilsson PJ, Ragnarsson-Olding BK. Importance of clear resection margins in anorectal malignant melanoma. Br J Surg. 2010;97:98-103.

18. Reina A, Errasti J, Espín E. Melanoma anorrectal. Cir Esp. 2014;92:510-56.

19. Matsuda K, Takifuji K, Hotta T, Yokoyama S; Ieda J; Iwamoto $\mathrm{H}$, et al. Laparoscopic abdominoperineal resection with lateral lymph node dissection for anorectal melanoma: A case report. Asian J Endosc Surg. 2016;9:65-7.

20. Bello DM, Smyth E, Pérez D, Khan S, Temple LK, Ariyan Ch, et al. Anal versus rectal melanoma: Does site of origin predict outcome? Dis Colon Rectum. 2013;56:150-7.

21. Diwan AK, Nangia N. Malignant melanoma. An exceptional survival of five years. Int J Med Res Prof. 2015;1:71-5.

Correspondencia: David Martínez-Ramos, MD Correo electrónico: doctormartinezramos@gmail.com Castellón, España. 